\title{
LOS CICLOS DE LOS PRECIOS DE LOS COMMODITIES Y SUS IMPACTOS MACRO-FINANCIEROS: EL CASO DEL PERÚ
}

\section{MARIO ZAMBRANO BERENDSOHN}

- Profesor de Administración de Riesgos

Carrera de Administración y Finanzas

Facultad de Negocios, UPC

\section{INTRODUCCIÓN}

\begin{abstract}
Este estudio realiza el análisis de los impactos macro-financieros de la dinámica de los precios de los commodities en un grupo de países, resaltándose el caso del Perú como exportador de minerales. En el periodo revisado se hace énfasis en el súper ciclo de los precios de los commodities que se inició por el 2004, que muestra en lo más reciente unos términos de intercambio que permanecen relativamente altos para los países commodity-exporters.
\end{abstract}

La fase creciente del súper ciclo ha tenido dos importantes efectos macroeconómicos en la muestra de países. Uno, el impulso que recibió el PBI desde el 2004 y el segundo, sobre la tasa de inversión en la minería, que fue extremadamente mejorada durante el boom de los commodities.

En el primer semestre de 2015 el índice de precios de los minerales se ha mantenido a la baja, como es la tendencia de los últimos años. Esto principalmente debido a caídas en los precios del cobre y del oro, que estarían regresando a sus fundamentos, impulsadas por la menor tasa de crecimiento de China, que incluye el ajuste de su mercado de valores; $y$ de una posible alza efectiva de la tasa de interés en la economía estadounidense estimado para el segundo semestre de 2015. En el caso del Perú, un hecho particular que hace la distinción en el análisis es el mayor grado de dolarización de su sistema financiero respecto a otros países commodity-exporters de la región. Ello puede asociarse, entre otros, a las diferencias en el ajuste cambiario 2014-15 que se ha venido observando en estas economías para reducir déficits de la cuenta corriente. La devaluación observada ha tenido ya consecuencias no sólo a través de la cuenta diferencia de cambio en los estados financieros de empresas, con mayor énfasis en aquellas empresas no exportadoras ni bancarias que fueron importantes emisores de deuda corporativa en el exterior en el periodo 2011-14, sino también con el incremento de la morosidad del sistema bancario.

En la parte final del estudio se discuten algunas recomendaciones de políticas macro-financieras que, para el caso del Perú, se podrían explorar para paliar los impactos macro-económicos del ajuste de precios del cobre.

\section{ANTECEDENTES}

\section{El súper ciclo de los commodities}

El crecimiento a tasas elevadas de economías como China, entre otras, en primer término y la crisis de confianza en varios países desarrolladas post crisis 2008 que implementaron una serie de programas de estímulos para reactivar el crecimiento económico, han favorecido a que los precios de los commodities se incrementen en valor. Así se inicia un gran súper ciclo en 2004 para los minerales y a fines de 2010 para el beef (carne de res), como se aprecia en la figura 1.

Figura 1. Indice de precios de minerales y beef $(2005=100)$

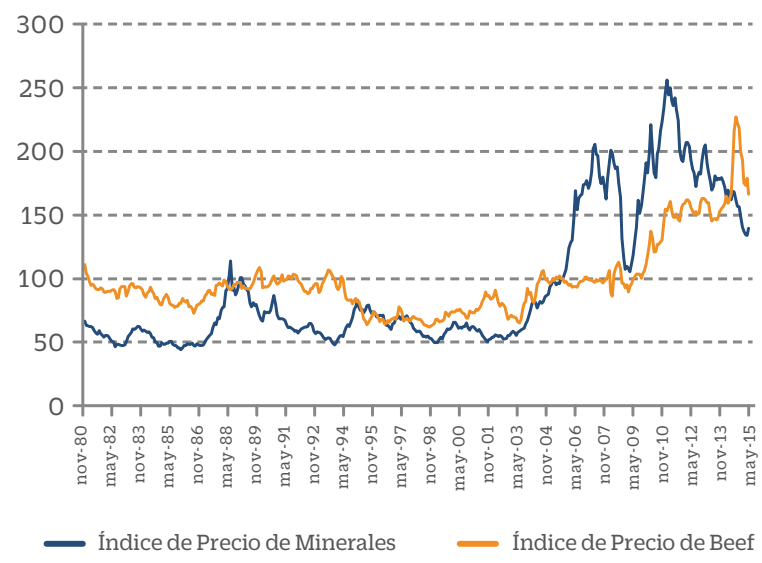

Fuente: $F M I / B M$

\section{El súper ciclo de los commodities y los países commodity exporters}

El súper ciclo de los precios de los commodities se inició por el 2004, mejorando los términos de intercambio, los mismos que aún están relativamente altos para los países commodity exporters (ver Figura 2). Después de su "peak", febrero 2011, estos han venido cayendo debido 
principalmente a la caída del cobre que ha caído más del $40 \%$ y el del petróleo que ha caído más del 50\% desde marzo 2012, como se aprecia en la Figura 3.

Figura 2. Términos de intercambio, países seleccionados (Índice, Febrero $00=100$ )

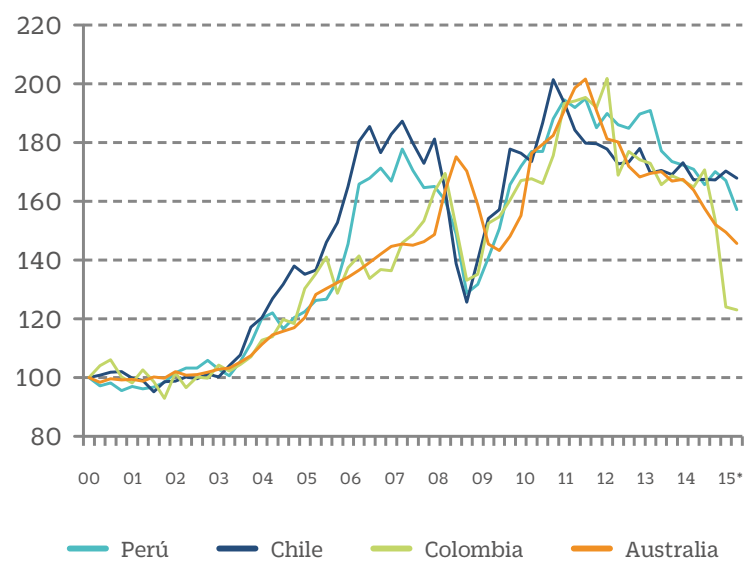

Figura 3.Precio de los commodities selectos (Índice, Marzo 09=100)

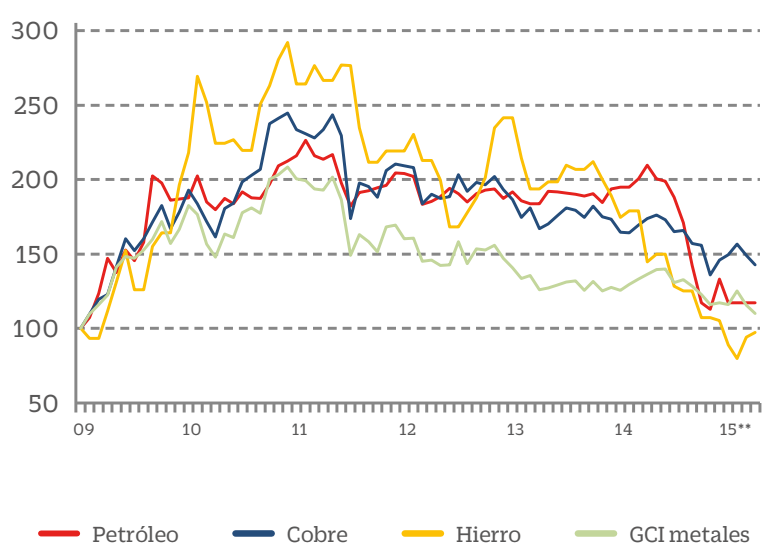

Fuente: Bloomberg , figura 2-3

(**) Al 1T - 2015
El boom de los precios de los commodity tuvo importantes efectos macroeconómicos, destacando dos: primero, el impulso que recibió el PBI desde el 2004, que después de la crisis 2008-09 tuvo como fundamento el fuerte impulso macroeconómico de China. Esto se visualiza en la tabla 1; el segundo efecto, sobre la tasa de inversión en la minería, que fue extremadamente mejorada durante el boom de los commodities y que también tuvo fuertes efectos multiplicadores sobre la inversión de los otros sectores no mineros, donde la inversión total explica aproximadamente el 30\% del producto. Lo anterior se muestra en la figura 4.

Tabla 1. Crecimiento del PBI para países seleccionados

\begin{tabular}{|c|c|c|c|c|c|c|}
\hline & Chile & Perú & Australia & Colombia & EE.UU & $\begin{array}{c}\text { Mundo } \\
\text { (Bloomberg) } \\
\left(^{*}\right)\end{array}$ \\
\hline 2014 & 1.8 & 2.4 & 2.4 & 4.6 & 2.4 & 2.5 \\
\hline $\begin{array}{c}\text { Average } \\
90-03\end{array}$ & 5.7 & 3.2 & 3.3 & 3.0 & 3.0 & 2.8 \\
\hline $\begin{array}{c}\text { Average } \\
04-13\end{array}$ & 4.8 & 6.4 & 3.0 & 4.8 & 1.7 & 2.5 \\
\hline
\end{tabular}

(*) Bloomberg only provides world growth record since 1996 Fuente: Bloomberg

Figura 4. Tasa de inversión minera y no minera Inversión Minera (\%PBI)

Inversión Minera (\%PBI)

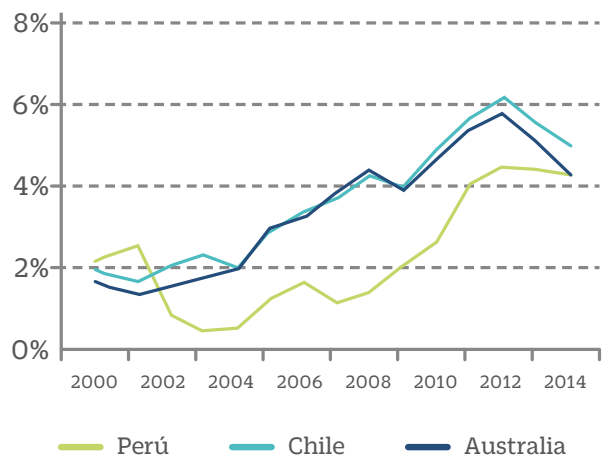

Bloomberg y Banco Central de cada país. 


\section{Inversión no-Minera}

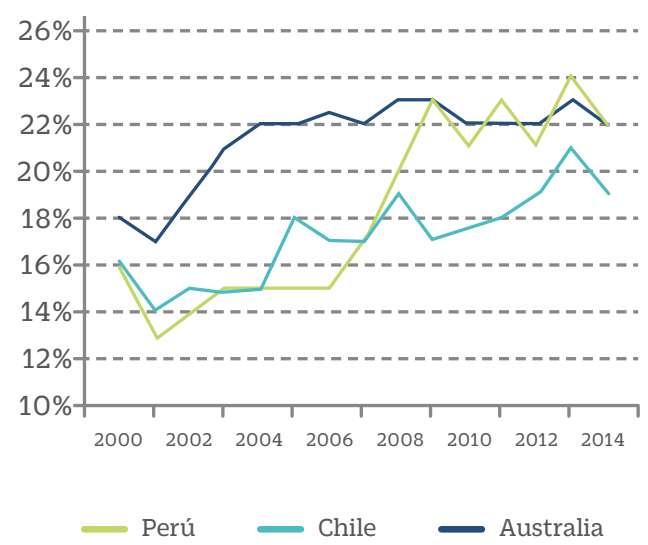

Bloomberg y Banco Central de cada país.

Así como se generó el proceso virtuoso en la fase del boom, ahora con la debilidad del crecimiento global, destacando China, se ha observado una fuerte caída en la tasa de inversión de la minería en los principales países exportadores de metales industriales, incluyendo Chile, Australia y el Perú. Por ejemplo, se hacen estimaciones de una caída de la inversión minera entre 15 al 18\% para el Perú en el 2015.

El boom de la inversión condujo a un progresivo deterioro del déficit de la cuenta corriente desde 2004, al principio con superávits que revelaban el esfuerzo fiscal de ahorrar parte de los mayores ingresos. En el 2013, Chile, Perú y Colombia registraban déficits de 3.7\%, 4.4\%, y 3.2\% del PBI, respectivamente. Ver Figuras $\mathbf{5}$ y $\mathbf{6}$.

Figura 5.Balanza de cuenta corriente, países seleccionados 2003-2015* (\%PBI)

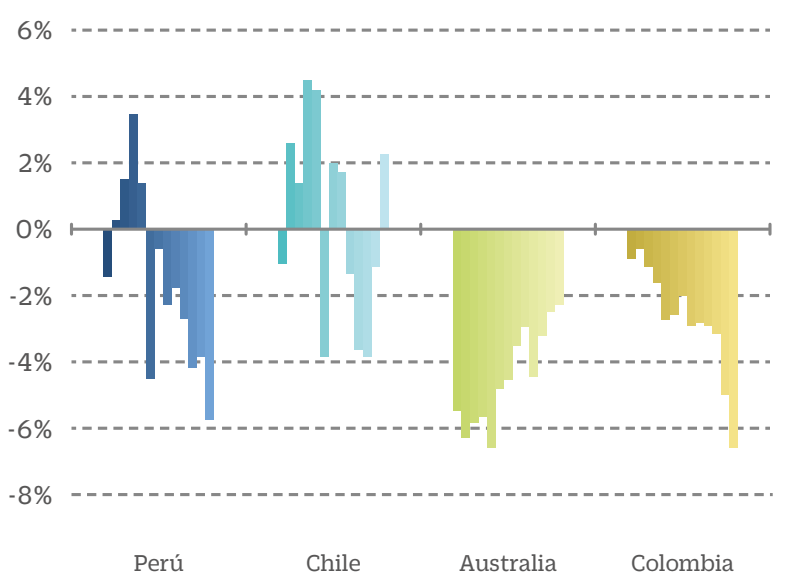

Figura 6.Balanza fiscal, países seleccionados 2003 2015* (\%PBI)

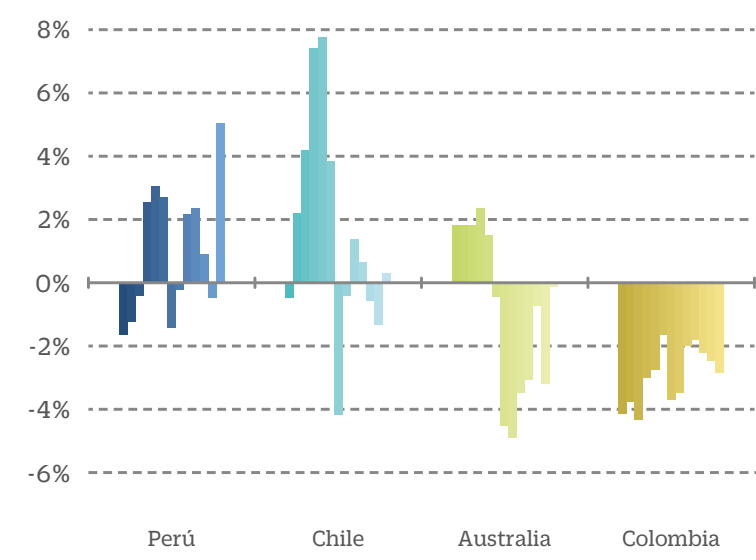

Fuente: Bancos centrales, figuras 5-6

(*) Al 1T - 2015

En periodo de auge de los precios, 2004-11, los mayores ingresos por exportaciones permitieron observar un escenario de apreciación en los años siguientes a la crisis de 2008. Para luego, desde 2013 iniciar un escenario de depreciación debido a la menor demanda de materias primas por parte de China, entre otros, y al anuncio del cierre del programa de estímulo monetario y la subida de tasas en los Estados Unidos.

La menor demanda agregada habría generado los deterioros en la cuenta corriente para la mayoría de los países, que se ajustó con una pronunciada depreciación del tipo de cambio. Esto se dio también en el Perú pero en menor medida, debido a la intervención sostenida en el mercado cambiario de parte del Banco Central, como se observa en las figuras $\mathbf{7}$ y 8 .

Figura 7. Episodios de apreciación, países seleccionados (Índice; Enero 04=100,Enero 10 = 100)

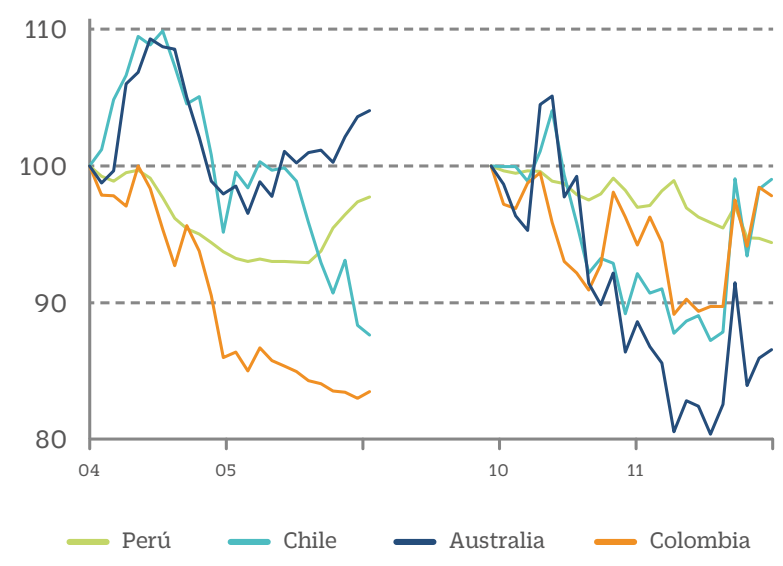


Figura 8. Eepisodios de apreciación, países seleccionados (Índice; Enero 08=100,Enero 13=100)

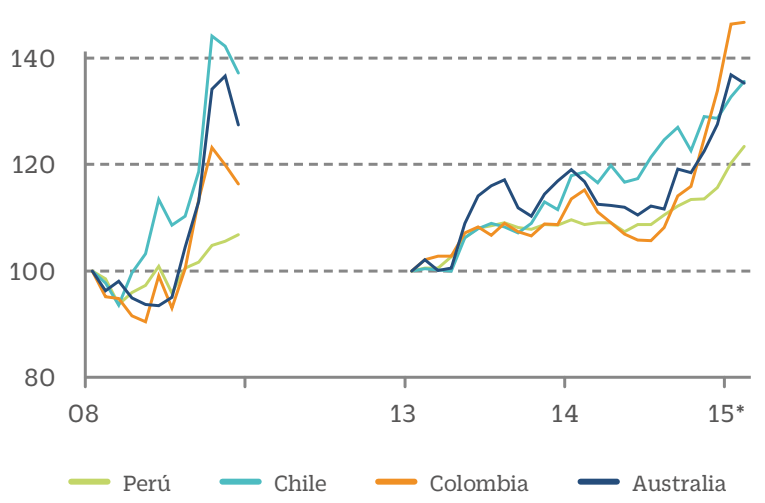

Fuente:Bloomberg, figura 7-8 (*) Al $2 \mathrm{~T}-2015$

\section{El súper ciclo de los precios de los commodities}

El índice de precios de los minerales se ha mantenido a la baja en el primer semestre de 2015. Esto principalmente a caídas en los precios del cobre y del oro, guiados por sus fundamentos, afectados por el menor crecimiento de economías como China, y al eventual alza de la tasa de interés de la economía estadounidense en el segundo semestre de 2015. Ver figuras 9 y 10.

Figura 9. Episodios de apreciación, países seleccionados (Índice; Enero 08=100,Enero 13=100)

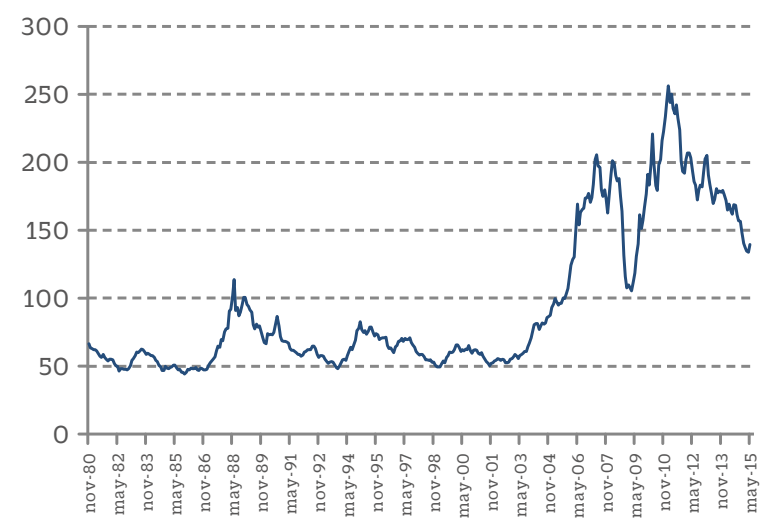

Fuente:FMI/BM

\section{Figura 10. Precio de commodities}

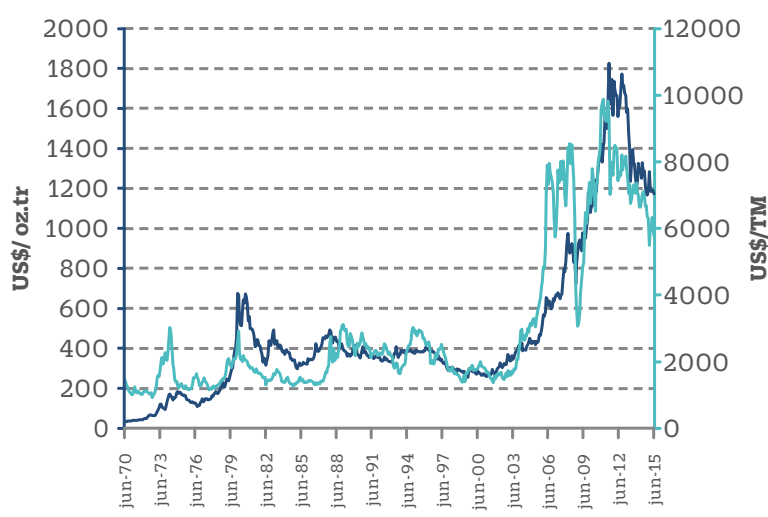

- Oro

- Cobre

Fuente:BM / Bloomberg

\section{Modelo del tipo de cambio nominal del Perú, Chile, Colombia}

Con la finalidad de estudiar el impacto cambiario por la dinámica de precios de commodities planteamos un modelo para el comportamiento del tipo de cambio, con los mismos fundamentos, para los países de Perú, Chile y Colombia, en función a su principal commodity de exportación respectivo.

El modelo es lineal en logaritmos y multivariable, con data diaria de enero de 2010 a julio de 2015. Se estima con mínimos cuadrados ordinarios. A continuación se muestra la forma funcional, además un cuadro con los coeficientes de las regresiones de los países antes mencionados.

$I T C=\beta_{0}+\beta_{1} \cdot$ lcommodity $+\beta_{2} \cdot I E M B I+\beta_{3} \cdot I D J+\beta_{4} \cdot I B R O A D$

donde:

- ITC: logaritmo del tipo de cambio nominal

- lcommodity: logaritmo del precio del principal commodity de exportación

- Lembi: logaritmo del embi (Indicador de Bonos de Mercados Emergentes)

- IDJ: logaritmo del índice del Dow Jones

- Lbroad: logaritmo del índice broad dollar 
Tabla $N^{\circ}$ 2. Coeficientes de la regresión estimada por el modelo

\begin{tabular}{|c|c|c|c|}
\hline Variable & Perú & Chile & Colombia \\
\hline Lcommodity & $-0.130^{* * *}$ & $-0.130^{* * *}$ & $-0.187^{* * *}$ \\
\hline lEMBI & $0.062^{* * *}$ & $0.033^{* * *}$ & $0.124^{* * *}$ \\
\hline lDJ & $-0.011^{* *}$ & $0.081^{* * *}$ & $0.186^{* * *}$ \\
\hline IBROAD & $0.341^{* * *}$ & $1.192^{* * *}$ & $0.574^{* * *}$ \\
\hline C & & $0.680^{* *}$ & $3.340^{* * *}$ \\
\hline$r^{2}$ & 0.999 & 0.851 & 0.908 \\
\hline \multicolumn{4}{|c|}{ leyenda: $p<0.05 ;{ }^{* *} p<0.01 ;{ }^{* * *} p<0.001$} \\
\hline Commodity & $\begin{array}{l}\text { Perú } \\
\text { cobre }\end{array}$ & $\begin{array}{l}\text { Chile } \\
\text { cobre }\end{array}$ & $\begin{array}{l}\text { Colombia } \\
\text { petróleo }\end{array}$ \\
\hline
\end{tabular}

Fuente: elaboración propia.

\section{ANÁLISIS DE RESULTADOS}

\section{Perú: Ciclo de commodities y rating}

En el caso del Perú, la fase positiva del ciclo de precios de los commodities se asocia a mejoras de sus rating, cuestión que revela la contingencia para la fase de ajuste de precios, como se aprecia en la figura 11.

\section{Figura 11. Precio de commodities para el Perú}

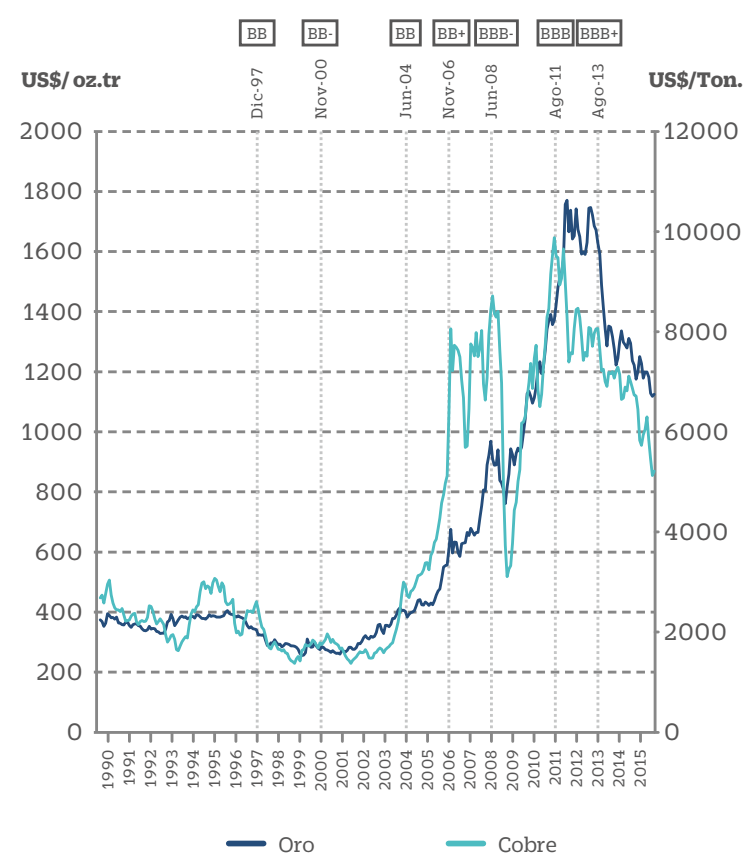

El rating crediticio del Perú ha venido mejorando en los últimos años, debido a la mejora en fundamentos económicos que también se asocian a un mayor flujo de ingresos debido a los altos precios de los commodities que exporta el país

La perspectiva del precio del cobre se centra principalmente en la China, como consecuencia de su crecimiento económico. Se infiere de la tabla 2, que sólo China representa aproximadamente el 50\% de la demanda mundial de este mineral, como se observa en las figura 12.

Figura 12. Variaciones anuales, PBI de China y cobre

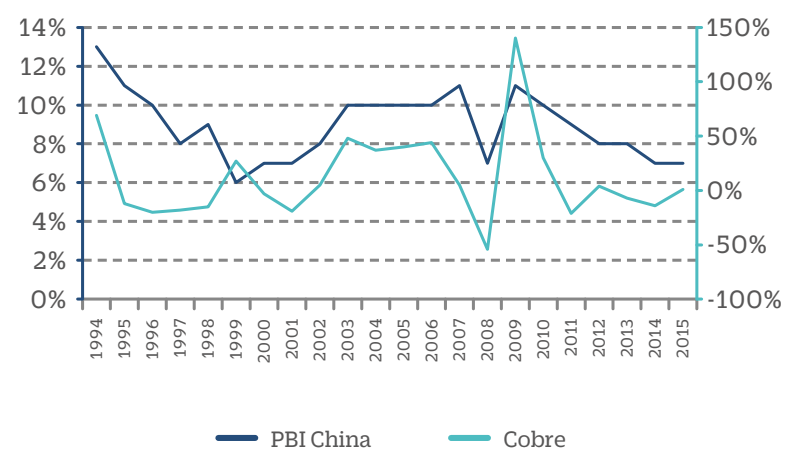

Fuente: Bloomberg , figura 11-12 (*) Al 2T - 2015

Tabla 3. Matriz de correlación, PBI China y cobre

\begin{tabular}{|c|c|c|}
\hline \multicolumn{1}{c|}{} & Cobre & PBI China \\
\hline Cobre & 1 & 0.56 \\
\hline PBI China & 0.56 & 1 \\
\hline
\end{tabular}

Fuente: Elaboración propia

\section{Ciclo de commodities y las exportaciones peruanas}

Hasta 2011, la participación de las exportaciones mineras en las exportaciones totales del país había crecido. De las exportaciones, las de cobre y del oro son las que han tenido una mayor participación. Esto se explica debido a la estructura productiva primario-exportador del país y a sus elevados precios hasta el 2011. Dada la coyuntura actual estas participaciones han venido disminuyendo, como se observa en las figuras 13-15. 
Figura 13. Participación de exportaciones en el PBI (precios corrientes)

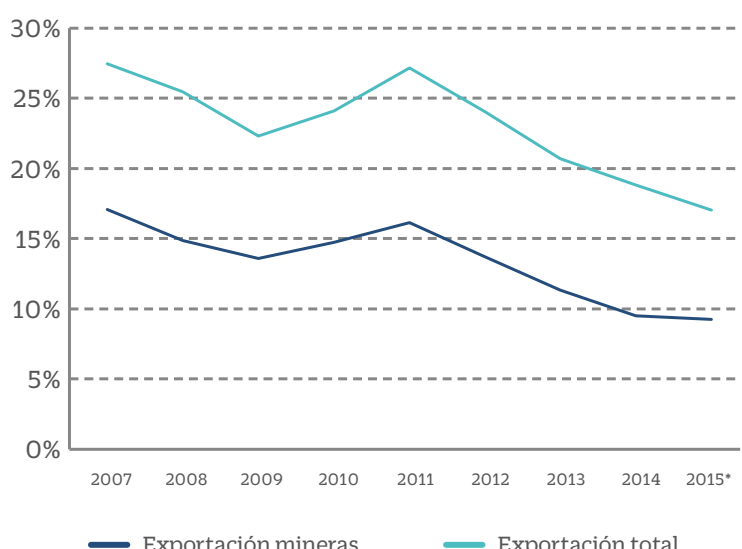

Fuente: SUNAT

(*) Al 1T - 2015

Figura 14. Participación de minerales en el PBI (precios corrientes)

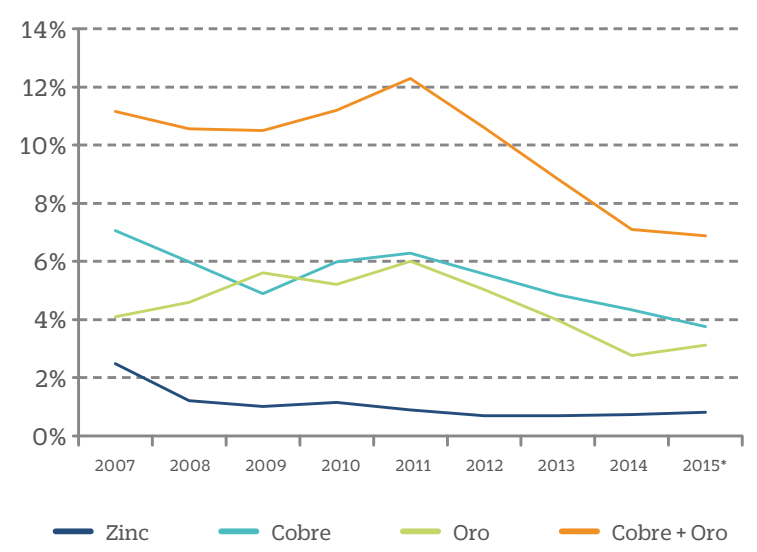

Fuente: SUNAT

(*) Al 1T - 2015
Figura 15. Participación de exportaciones mineras

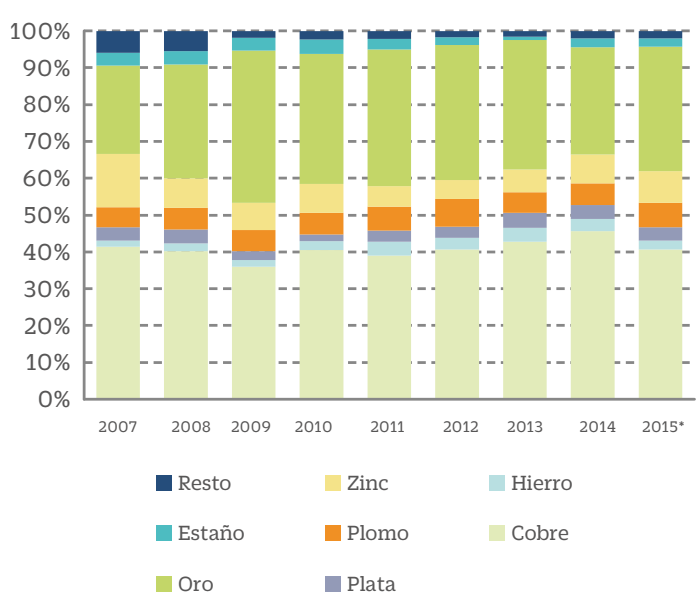

Fuente: SUNAT

(*) Al 1T - 2015

\section{Déficit, la cuenta corriente y el precio de los commodities}

El déficit en cuenta corriente de pagos muestra, en la figura 16, una relación negativa con la variación de precio del oro y del cobre. Mayores precios indican mayores ingresos al país y menor necesidad de financiamiento externo. Con la caída de precios de los últimos años el déficit de cuenta corriente ha aumentado y ha llegado a 5.1\% en el primer semestre del 2015.

Figura 16. Evolución del déficit de cuenta corriente y del precio de commodities

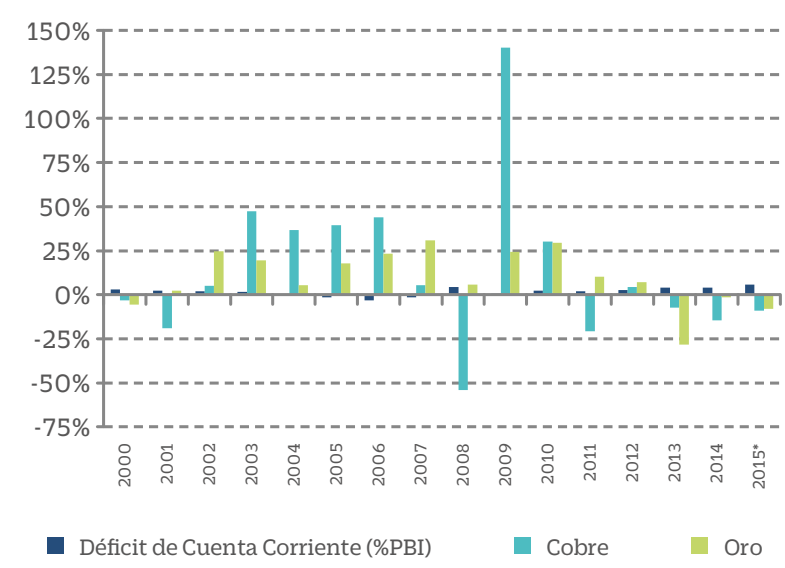

Fuente: Bloomberg / BCR

(*) Al 1T - 2015 


\section{SBVL, Commodities y PBI}

El ISBVL muestra un importante crecimiento acumulado hasta antes de la crisis de Lehman Brothers. Pasando luego a recuperarse y tener rendimientos positivos hasta el 2011, donde pasa a una fase de ajuste, asociada a su exposición al sector minero, es decir, al efecto precio. Lo anterior se aprecia en la figura 17.

Así mismo, habría evidencia que el índice se comportaría como un indicador adelantado del crecimiento del PBI, con una correlación de 0.62 entre el índice con la tasa de crecimiento del PBI y el ISBVL del año posterior, como se muestra en la tabla 4.

Figura 17. Variaciones anuales del PBI de Perú \& ISBVL

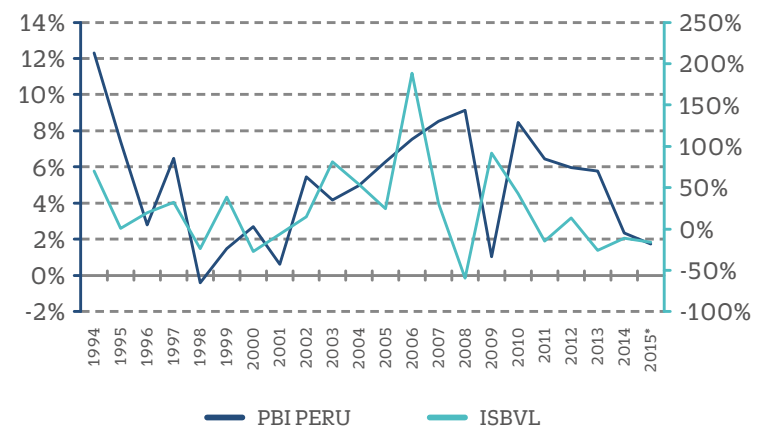

Fuente: Bloomberg / BCR

(*) Al 1T - 2015

Tabla 4. Matriz de correlaciones entre ISBVL y PBI Perú (en tasas, \%)

\begin{tabular}{|c|c|}
\hline & PBI Perú $(\mathrm{t})$ \\
\hline ISBVL $(\mathrm{t})$ & 0.20 \\
\cline { 1 - 2 } ISBVL ( $\mathrm{t}-1)$ & 0.62 \\
\hline
\end{tabular}

Fuente: Elaboración propia

Una relación similar se puede encontrar entre el índice selectivo de la bolsa de valores de Lima con los principales metales exportados por el país. Una correlación positiva que muestra que nuestro mercado de valores depende significativamente de las empresas productoras y exportadoras de estos metales, como se aprecia en la figura 18 y la tabla 5.
Figura 18. Variaciones anuales de ISBVL, Cobre y Oro.

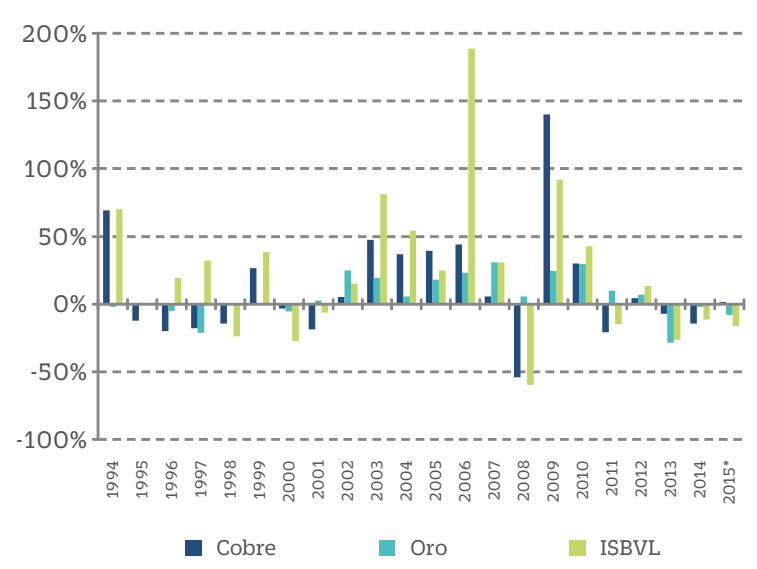

Fuente: Bloomberg / BCR

(*) Al 1T - 2015

Tabla 5. Crecimiento acumulado, Cobre, Oro \& ISBVL

\begin{tabular}{|c|c|c|c|} 
& $\begin{array}{c}1993- \\
08 / 2008\end{array}$ & $\begin{array}{c}09 / 2008- \\
2015^{*}\end{array}$ & $\begin{array}{c}1993- \\
2015^{*}\end{array}$ \\
\cline { 1 - 4 } Cobre & $320 \%$ & $-5 \%$ & $238 \%$ \\
\cline { 1 - 4 } Oro & $113 \%$ & $36 \%$ & $203 \%$ \\
\cline { 1 - 4 } ISBVL & $2185 \%$ & $-8 \%$ & $1661 \%$ \\
\hline
\end{tabular}

Fuente: Elaboración propia.

\section{Mercado de Emisiones y Rating: Riesgos Inherentes}

Dada la mejora en la calificación crediticia soberana, el país ha podido financiarse mediante emisiones locales y externas, tanto a nivel soberano como corporativo, principalmente en el mercado internacional en el periodo 2012-14. Ver la figura 19.

\section{Figura 19. Emisiones totales según mercado}

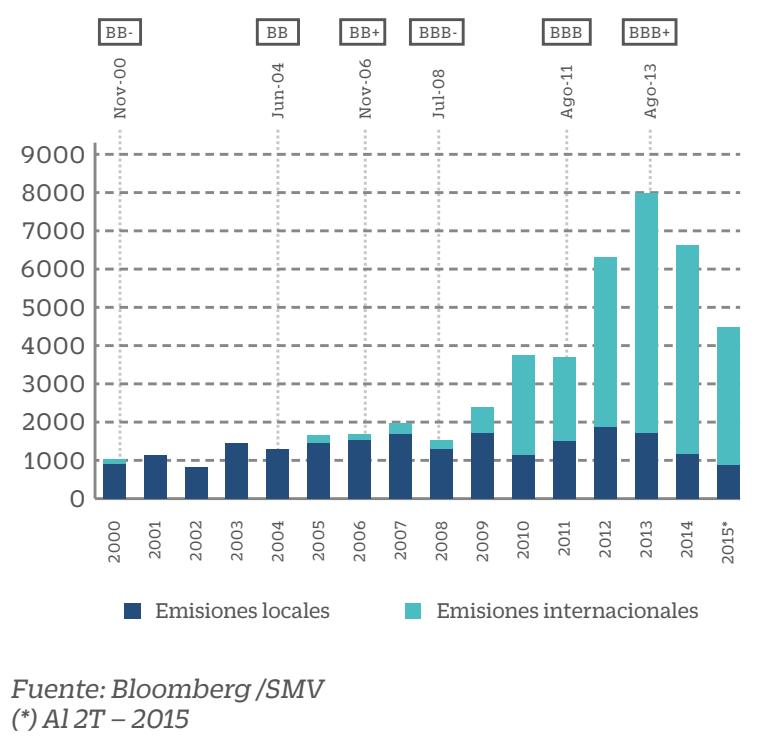


No obstante, la favorable condición de contar con el mercado externo de financiamiento disponible, a tasas menores, mayores duraciones y montos significativos para las empresas corporativas peruanas, tanto de grado de inversión, como de corte especulativo, dos hechos, como el menor crecimiento global y de la región, y los ajustes cambiarios observados, y que presumiblemente continuarán, están configurando un perfil de riesgos complicado para aquellos emisores ni exportadores ni bancarios que participaron en los mercados internacionales durante el 2011-14: el incremento relativo del riesgo de apalancamiento y la presencia de un material riesgo cambiario. Lo anterior se observa en la figura 20 y tabla 6.

Señalar también que este perfilamiento es un hecho empírico de varios países emergentes, es decir, la deuda corporativa emergente se encuentra actualmente en la matriz de riesgos relevantes para los reguladores, organismos financieros internacionales, y clasificadoras de riesgos. Así, entre el 2014 y lo que va del 2015 ya se han observado una serie de downgrades de rating en la región, que seguramente se profundizará al menos hasta el 2016. La realización de estos riesgos ya se viene observando a través de la caída de las utilidades de ls empresas listadas en bolsa y en los mayores gastos de la cuenta diferencia de cambio, que en algunas empresas significa una proporción importante de su ebitda o margen operativo.

Figura 20. Crecimiento en $\%$ anual, PBI y emisiones totales en circulación

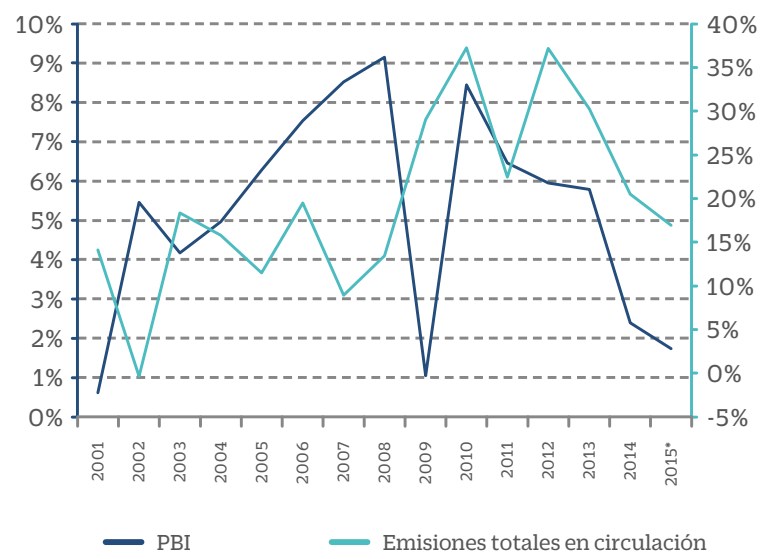

Fuente: Bloomberg /SMV

(*) Al $2 \mathrm{~T}-2015$

Tabla 6. Crecimiento acumulado, Cobre, Oro \& ISBVL

\begin{tabular}{|c|c|c|c|}
\hline$\triangle \mathrm{ETC}$ & $98 \%$ & $367 \%$ & $949 \%$ \\
\hline$\Delta \mathrm{PBI}$ & $43 \%$ & $34 \%$ & $109 \%$ \\
\hline $\begin{array}{l}\Delta \mathrm{ETC} / \\
\Delta \mathrm{PBI}\end{array}$ & 2.28 & 10.83 & 8.71 \\
\hline
\end{tabular}

Fuente: Elaboración propia.

Así como hubo un crecimiento elevado del financiamiento a través de la emisión de bonos, fundamentalmente externos, también se registraron tasas materiales de crecimiento a través del fondeo bancario, es decir, los créditos otorgados por la banca múltiple.

Para generar una medida comparativa de estos crecimientos de fondeo se usaron múltiplos en función al crecimiento del PBI, subdividiendo por periodos previos y post la crisis sub-prime. Los resultados presentados en la figura 21 y en la tabla 7 muestran para el periodo relevante, 2008-14, que el crecimiento de ambas fuentes ha sido varias veces mayor al del PBI lo que invita a estudiar mejor la situación de apalancamiento.

Figura 21. Crecimiento porcentual anual del PBI y créditos brutos

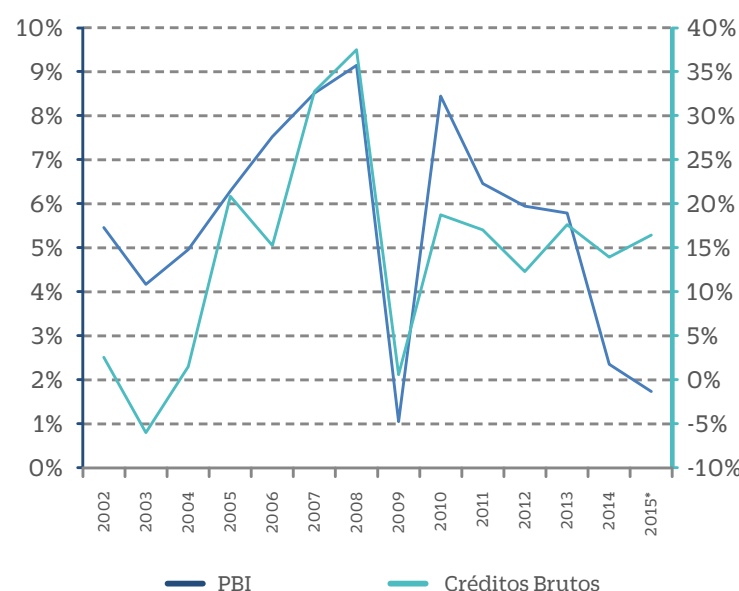

Fuente: $B C R / S B S$

Tabla 7. Crecimiento acumulado, créditos brutos y PBI

\begin{tabular}{|c|c|c|c|}
\hline & 2001-2007 & $2008-2014$ & 2001-2014 \\
\hline $\begin{array}{c}\Delta \text { Créditos } \\
\text { Brutos }\end{array}$ & $81 \%$ & $110 \%$ & $423 \%$ \\
\hline$\triangle \mathrm{PBI}$ & $43 \%$ & $34 \%$ & 109\% \\
\hline $\begin{array}{l}\Delta \mathrm{CB} / \\
\Delta \mathrm{PBI}\end{array}$ & 1.88 & 3.25 & 3.88 \\
\hline
\end{tabular}

Fuente: Elaboración propia. 


\section{Crecimiento del Fondeo, PBI y Commodities}

El crecimiento del FT (Funding Total aproximado por la suma de los saldos en circulación de emisiones y créditos brutos), ha tenido un desempeño correlacionado con el Crecimiento del PBI, y por ende con el ciclo de precios de los commodities, pero en los episodios más recientes se ha comenzado a generar una brecha, la que podría evidenciar un mayor apalancamiento. Lo anterior se evidencia en las figuras 22 y 23 y en la tabla 8 . La discusión que queda pendiente es si es sostenible el crecimiento del endeudamiento de las empresas con tasas menores de crecimiento del producto, variable que en términos gruesos mediría la capacidad de pagos o ingresos de la economía.

Figura 22. Crecimiento porcentual anual, PBI y Funding Total

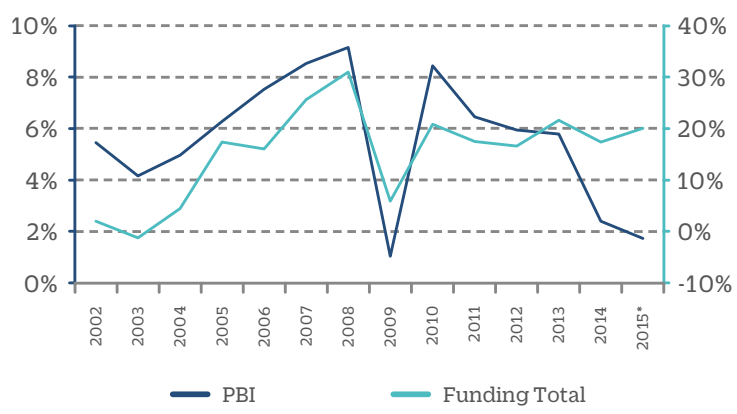

Fuente: $B C R / S B S$

(*) Al 1T - 2015

Tabla 8. Crecimiento acumulado, Funding Total y PBI

\section{1-2007 2008-2014 $2001-2014$}

\begin{tabular}{|c|c|c|c|}
\hline$\Delta \mathrm{FT}$ & $80 \%$ & $151 \%$ & $491 \%$ \\
\hline$\Delta \mathrm{PBI}$ & $43 \%$ & $34 \%$ & $109 \%$ \\
\hline$\Delta \mathrm{FT} /$ & 1.86 & 4.44 & 4.51 \\
\hline
\end{tabular}

Fuente: Elaboración propia.

\section{Figura 23. Funding y cobre}

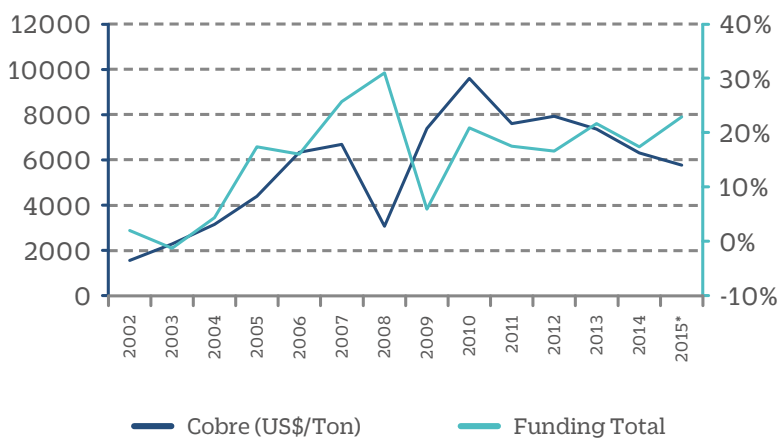

Fuente: $B C R / S B S /$ Bloomberg

(*) Al 2T-2015

\section{Retail y Endeudamiento}

Dada la correlación entre la inversión minera y la inversión en otros sectores económicos, y su impacto en el crecimiento del país, no sólo se ha observado el aumento del fondeo total comercial y corporativo sino también a nivel minorista o también llamado "retail", el cual ha sido uno de los pilares del sostenimiento del consumo. Ver figura 24.

\section{Figura 24. Crecimientos anuales, líneas de crédito}

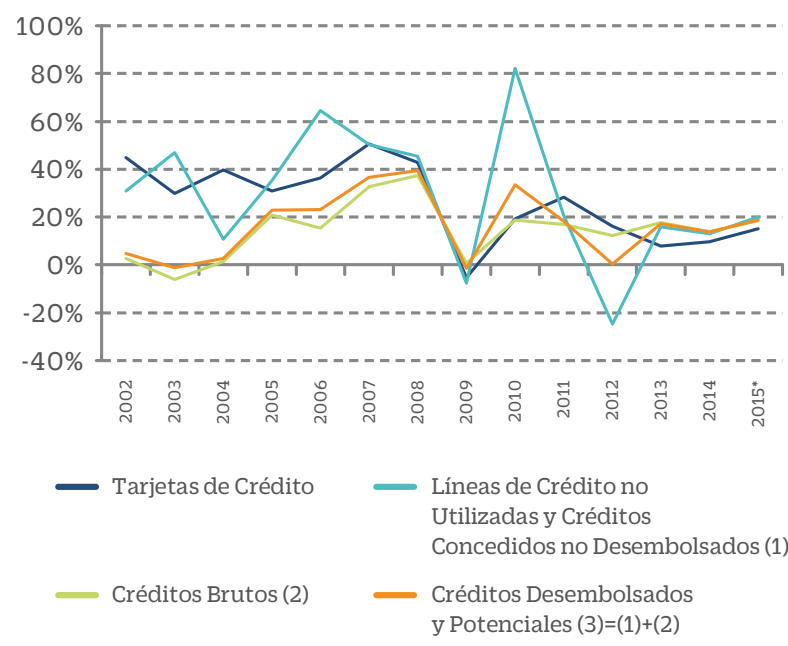

Fuente: $S B S$

(*)A mayo 2015 
Así, la evidencia empírica muestra, por ejemplo, una ratio de crecimiento acumulado de tarjetas de crédito sobre el crecimiento del PBI de 18 veces para todo el periodo analizado, como se aprecia en la tabla 9.

Tabla 9. Ratios de crecimiento acumulado, Créditos y PBI

\begin{tabular}{|c|c|}
\hline$\Delta$ Tarjeta Crédito/ $\triangle \mathrm{PBI}$ & 18 \\
\hline$\Delta$ Créditos Brutos/ $\Delta \mathrm{PBI}$ & 4 \\
\hline$\Delta$ Créditos D y P/APBI & 5
\end{tabular}

Fuente: Elaboración propia.

Si bien los múltiplos más elevados se dieron antes de la crisis financiera sub prime, los múltiplos correspondientes al segmento de post crisis 2008 mostrarían que el mayor apalancamiento también pudiera estar afectando a las carteras minoristas del sistema financiero - ver tabla 10.

Tabla 10. Ratios de crecimiento acumulado Créditos y PBI, 2001-2007 y 2008-2014

\begin{tabular}{|c|c|}
\hline & 2001-2007 \\
\hline$\Delta$ Tarjeta Crédito/ $\triangle \mathrm{PBI}$ & 14 \\
\hline$\Delta$ Créditos Brutos/ $\triangle \mathrm{PBI}$ & 2 \\
\hline$\Delta$ Créditos $\mathrm{D}$ y $\mathrm{P} / \Delta \mathrm{PBI}$ & 3 \\
\hline & 2008-2014 \\
\hline$\Delta$ Tarjeta Crédito/ $\triangle \mathrm{PBI}$ & 3 \\
\hline$\Delta$ Créditos Brutos/ $\Delta \mathrm{PBI}$ & 3 \\
\hline$\Delta$ Créditos D y $\mathrm{P} / \Delta \mathrm{PBI}$ & 3 \\
\hline
\end{tabular}

Fuente: Elaboración propia.

\section{CONCLUSIONES: ¿CICLO ECONÓMICO O FUNDAMENTALES?}

La influencia externa es evidente. No sólo el tema del "tapering" sino lo que pase con la demanda externa desde China, que puede entrar en una mini crisis y/o crecer menos.

El precio de los minerales, básicamente del cobre, impacta en el mantenimiento del déficit en cuenta corriente (CC). Otros países han devaluado, notoriamente más que el Perú para cerrar ese desequilibrio externo. De mantenerse este déficit pudiera afectar, entre otros,al rating soberano.

El mercado de bonos corporativos ha sido básicamente de emisiones externas en 2011-14, por lo que hay exposición al riesgo cambiario y en menor medida al crediticio. De hecho, empresas de otros países de Latinoamérica ya han caído en problemas recientemente, incluyendo downgrades de bonos corporativos peruanos.

Al tener una parcial dolarización crediticia, la corrección del déficit de la CC, vía ajustes cambiarios, tendrá impacto en la morosidad.

En el análisis de oferta y demanda del financiamiento, se observa que los oferentes crediticios sostienen una posición de liquidez y fondos prestables al menos para el periodo analizado, mientras que los sujetos de crédito pueden haber visto afectada su capacidad de pago por el menor crecimiento y la subida del tipo de cambio, aunado a las incertidumbres externa e interna.

Las medidas de política financiera dictadas por las autoridades han estado orientadas, básicamente, hacia dar liquidez (encaje y repos), y flexibilizar en provisiones y capital, ante expansión crediticia, a los oferentes de créditos.

Es decir, el tema de fondo para ser competitivo pasa por medidas para potenciar la capacidad de pago: inversión y consumo. Luego, las políticas a evaluar pasarían, por ejemplo, por rebajas del IGV, hacer un mayor uso de las emisiones soberanas, ajustes y reforma en el sistema privado de pensiones para permitir uso de fondos "en exceso" para adquisición de activos de largo plazo y pago de deuda hipotecaria, etc.

Es decir, buscar potenciar la demanda interna.

Finalmente, nuestra dependencia estructural hacia los commodities debería llevar a evaluar el fondo que en un principio se generó con las privatizaciones. A diferencia del Chile, donde hay un fondo de estabilización basado en los mayores precios del cobre, en Perú habría que repensar ese "buffer" en la medida en que la actividad minera es fundamentalmente privada. 


\section{REFERENCIAS BIBLIOGRÁFICAS}

Cowan, K., Rappoport, D. \& Selaive, J. (2007). High

Frequency Dynamics of the Exchange Rate in Chile.

Banco Central de Chile.

Cashin, P., Céspedes L. \& Sahay R. (2004). Commodity

Currencies and the Real Exchange Rate. Journal of

Development Economics.

Claro, S. (2015). Macroeconomic Adjustment in the Andean Countries to the End of the Commodity Super Cycle. Banco Central de Chile.

Moreno C., Saavedra C. \& Ulloa, B. (2014).

Commodity Price Cycles and Financial Stability.

Banco Central de Chile.

Yi Wu (2013). What Explains Movements in the Peso/

Dollar Exchange Rate?. Fondo Monetario Internacional. 


\section{ANEXO1:}

Matrices de correlaciones y resumen estadístico. Matrices de Correlaciones enero 2000 - junio 2015

\begin{tabular}{|c|c|c|c|c|c|}
\hline & Oro & Cobre & Petróleo & IGBVL & ISBVL $^{* *}$ \\
\hline Oro & 1 & 0,30 & 0,27 & 0,32 & 0,32 \\
\hline Cobre & 0,30 & 1 & 0,43 & 0,55 & 0,55 \\
\hline Petróleo & 0,27 & 0,43 & 1 & 0,39 & 0,39 \\
\hline IGBVL & 0,32 & 0,55 & 0,39 & 1 & 0,98 \\
\hline ISBVL $^{* *}$ & 0,32 & 0,55 & 0,39 & 0,98 & 1 \\
\hline
\end{tabular}

Fuente: Bloomberg

$\left.{ }^{* *}\right)$ A abril 2015

\section{MATRICES 2000 - 2008}

Matrices de Correlaciones 2000-2008

\begin{tabular}{|c|c|c|c|c|c|c|}
\hline & TC & Oro & Cobre & Petróleo & IGBVL & ISBVL \\
\hline TC & 1 & $-0,17$ & $-0,09$ & 0,04 & $-0,13$ & $-0,11$ \\
\hline Oro & $-0,17$ & 1 & 0,36 & 0,26 & 0,22 & 0,21 \\
\hline Cobre & $-0,09$ & $-0,36$ & 1 & 0,2 & 0,42 & 0,44 \\
\hline Petróleo & 0,04 & 0,26 & 0,2 & 1 & 0,17 & 0,18 \\
\hline IGBVL & $-0,13$ & 0,22 & 0,42 & 0,17 & 1 & 0,98 \\
\hline ISBVL & $-0,11$ & 0,21 & 0,44 & 0,18 & 0,98 & 1 \\
\hline
\end{tabular}

Fuente: Bloomberg

Matrices de Correlaciones 2000-2008 (Enero 2000 - Agosto 2008)

\begin{tabular}{|c|c|c|c|c|c|c|c|c|c|}
\hline & TC & Oro & Cobre & Petróleo & IGBVL & ISBVL & Dow Jones & Shangai & Zinc \\
\hline TC & 1 & $-0,17$ & $-0,09$ & 0,04 & $-0,13$ & $-0,11$ & $-0,07$ & $-0,08$ & $-0,07$ \\
\hline Oro & $-0,17$ & 1 & 0,36 & 0,26 & 0,22 & 0,21 & $-0,13$ & 0,15 & 0,24 \\
\hline Cobre & $-0,09$ & $-0,36$ & 1 & 0,2 & 0,42 & 0,44 & 0,24 & 0,18 & 0,67 \\
\hline Petróleo & 0,04 & 0,26 & 0,2 & 1 & 0,17 & 0,18 & $-0,14$ & 0,13 & 0,10 \\
\hline IGBVL & $-0,13$ & 0,22 & 0,42 & 0,17 & 1 & 0,98 & 0,29 & 0,32 & 0,34 \\
\hline ISBVL & $-0,11$ & 0,21 & 0,44 & 0,18 & 0,98 & 1 & 0,29 & 0,32 & 0,37 \\
\hline Dow Jones & $-0,07$ & $-0,13$ & 0,24 & $-0,14$ & 0,29 & 0,29 & 1 & 0,20 & 0,24 \\
\hline Shangai & $-0,08$ & 0,15 & 0,18 & 0,13 & 0,32 & 0,32 & 0,20 & 1 & 0,29 \\
\hline Zinc & $-0,07$ & 0,24 & 0,67 & 0,10 & 0,34 & 0,37 & 0,24 & 0,29 & 1 \\
\hline
\end{tabular}

Fuente: Bloomberg 
RESUMEN ESTADÍSTICO 2000-2008 (ENERO 2000 - AGOSTO 2008)

\begin{tabular}{|c|c|}
\hline \multicolumn{2}{|l|}{ ORO } \\
\hline Media & 0,011 \\
\hline Error típico & 0,004 \\
\hline Mediana & 0,013 \\
\hline Moda & - \\
\hline Desviación estándar & 0,043 \\
\hline Varianza de la muestra & 0,002 \\
\hline Curtosis & $-0,105$ \\
\hline Coeficiente de asimetría & 0,214 \\
\hline Rango & 0,214 \\
\hline Mínimo & $-9,3 \%$ \\
\hline Máximo & $12,1 \%$ \\
\hline Suma & 1,173 \\
\hline Cuenta & 103 \\
\hline Mayor (1) & $12,1 \%$ \\
\hline Menor (1) & $-9,3 \%$ \\
\hline $\begin{array}{c}\text { Nivel de confianza } \\
(99.0 \%)\end{array}$ & 0,011 \\
\hline
\end{tabular}

\begin{tabular}{|c|c|}
\hline \multicolumn{2}{|l|}{ COBRE } \\
\hline Media & 0,016 \\
\hline Error típico & 0,007 \\
\hline Mediana & 0,01 \\
\hline Moda & - \\
\hline Desviación estándar & 0,071 \\
\hline Varianza de la muestra & 0,005 \\
\hline Curtosis & 1,293 \\
\hline Coeficiente de asimetría & 0,79 \\
\hline Rango & 0,407 \\
\hline Mínimo & $-11,0 \%$ \\
\hline Máximo & $29,7 \%$ \\
\hline Suma & 1,646 \\
\hline Cuenta & 103 \\
\hline Mayor (1) & $29,7 \%$ \\
\hline Menor (1) & $-11,0 \%$ \\
\hline $\begin{array}{l}\text { Nivel de confianza } \\
(99.0 \%)\end{array}$ & 0,018 \\
\hline
\end{tabular}

Fuente: Bloomberg

\section{MATRICES 2008 - 2015}

Matrices de Correlaciones 2008-2015 (Setiembre 2008 - Junio 2015)

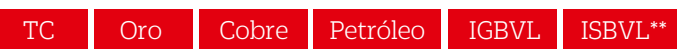

\begin{tabular}{|c|c|c|c|c|c|c|c|}
\hline TC & 1 & $-0,17$ & $-0,47$ & $-0,28$ & $-0,47$ & $-0,49$ \\
\cline { 1 - 5 } Oro & $-0,17$ & 1 & 0,25 & 0,27 & 0,38 & 0,40 \\
\hline Cobre & $-0,47$ & 0,25 & 1 & 0,67 & 0,65 & 0,64 \\
\hline Petróleo & $-0,28$ & 0,27 & 0,67 & 1 & 0,58 & 0,58 \\
\hline IGBVL & $-0,47$ & 0,38 & 0,65 & 0,58 & 1 & 0,99 \\
\hline ISBVL & $-0,49$ & 0,40 & 0,64 & 0,58 & 0,99 & 1 \\
\hline
\end{tabular}

Fuente: Bloomberg / (**) A abril 2015 
Matrices de Correlaciones 2008-2015 (Setiembre 2008 - Junio 2015)

\begin{tabular}{|c|c|c|c|c|c|c|c|c|c|}
\hline & TC & Oro & Cobre & Petróleo & IGBVL & ISBVL & Dow Jones & Shangai & Zinc \\
\hline TC & 1 & $-0,17$ & $-0,47$ & $-0,28$ & $-0,47$ & $-0,49$ & $-0,47$ & $-0,05$ & $-0,38$ \\
\hline Oro & $-0,17$ & 1 & 0,25 & 0,27 & 0,38 & 0,40 & 0,11 & 0,19 & 0,35 \\
\hline Cobre & $-0,47$ & 0,25 & 1 & 0,67 & 0,65 & 0,64 & 0,65 & 0,46 & 0,79 \\
\hline Petróleo & $-0,28$ & 0,27 & 0,67 & 1 & 0,58 & 0,58 & 0,55 & 0,26 & 0,52 \\
\hline IGBVL & $-0,47$ & 0,38 & 0,65 & 0,58 & 1 & 0,99 & 0,49 & 0,36 & 0,63 \\
\hline ISBVL & $-0,49$ & 0,40 & 0,64 & 0,58 & 0,99 & 1 & 0,49 & 0,38 & 0,65 \\
\hline Dow Jones & $-0,47$ & 0,11 & 0,65 & 0,65 & 0,49 & 1 & 0,49 & 0,32 & 0,60 \\
\hline Shangai & $-0,05$ & 0,19 & 0,46 & 0,26 & 0,36 & 0,38 & 0,31 & 1 & 0,53 \\
\hline Zinc & $-0,38$ & 0,35 & 0,79 & 0,52 & 0,63 & 0,65 & 0,60 & 0,53 & 1 \\
\hline
\end{tabular}

Fuente: Bloomberg / (*) A abril 2015

Resumen Estadístico 2008-2015 (Setiembre 2008 - Junio 2015)

\begin{tabular}{|c|c|}
\hline \multicolumn{2}{|l|}{ ORO } \\
\hline Media & 0,006 \\
\hline Error típico & 0,006 \\
\hline Mediana & 0,004 \\
\hline Moda & - \\
\hline Desviación estándar & 0,058 \\
\hline Varianza de la muestra & 0,003 \\
\hline Curtosis & 0,250 \\
\hline Coeficiente de asimetría & $-0,153$ \\
\hline Rango & 0,299 \\
\hline Mínimo & $-16,9 \%$ \\
\hline Máximo & $13,0 \%$ \\
\hline Suma & 0,480 \\
\hline Cuenta & 82 \\
\hline Mayor (1) & $13,0 \%$ \\
\hline Menor (1) & $-16,9 \%$ \\
\hline $\begin{array}{c}\text { Nivel de confianza } \\
(99.0 \%)\end{array}$ & 0,013 \\
\hline
\end{tabular}

\begin{tabular}{|c|c|}
\hline \multicolumn{2}{|l|}{ COBRE } \\
\hline Media & 0 \\
\hline Error típico & 0,009 \\
\hline Mediana & 0,011 \\
\hline Moda & - \\
\hline Desviación estándar & 0,083 \\
\hline Varianza de la muestra & 0,007 \\
\hline Curtosis & 3,946 \\
\hline Coeficiente de asimetría & $-1,131$ \\
\hline Rango & 0,527 \\
\hline Mínimo & $-35,6 \%$ \\
\hline Máximo & $17,1 \%$ \\
\hline Suma & 0,037 \\
\hline Cuenta & 82 \\
\hline Mayor (1) & $17,1 \%$ \\
\hline Menor (1) & $-35,6 \%$ \\
\hline $\begin{array}{l}\text { Nivel de confianza } \\
(99.0 \%)\end{array}$ & 0,018 \\
\hline
\end{tabular}




\section{ANEX02:}

Regresión del tipo de cambio

$$
\text { ITC }=\beta_{0}+\beta_{1}{ }^{*} \text { Icommodity }+\beta_{2}{ }^{*} I E M B I+\beta_{3}{ }^{*} I D J+\beta_{4}{ }^{*} I B R O A D
$$

\section{PAÍSES}

\section{PERÚ}

. Reg lTC_PER ICOBRE IEMBI_PER IDJ IBROAD, r nOC

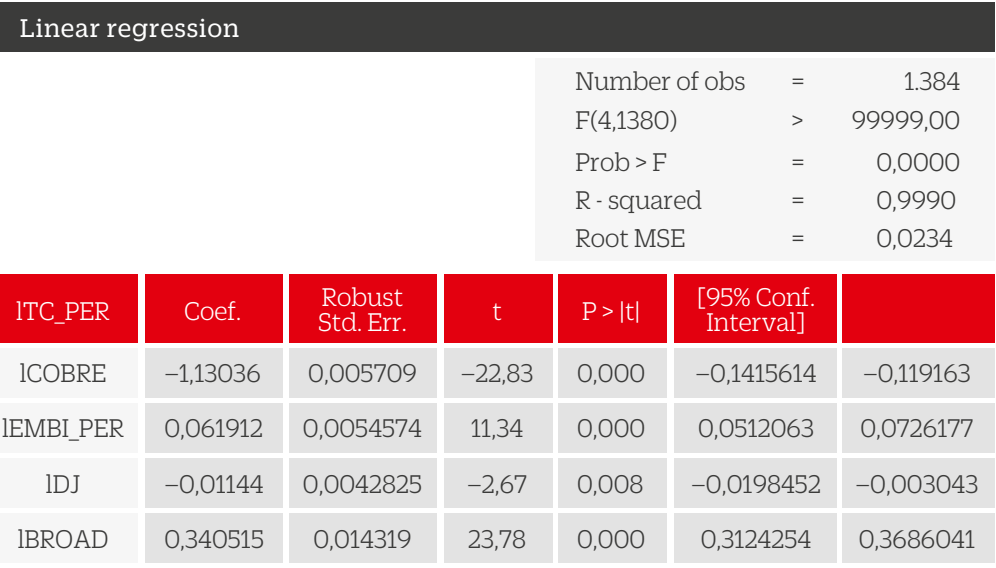


CHILE

.Reg lTC_CHI lCOBRE IEMBI_CHI IDJ IBROAD, r

Linear regression

\begin{tabular}{|c|c|c|c|c|c|c|c|}
\hline & & & & \multicolumn{2}{|c|}{ Number of obs } & $=$ & 1.384 \\
\hline & & & & \multicolumn{2}{|c|}{$F(4,1379)$} & $>$ & 4836,35 \\
\hline & & & & \multicolumn{2}{|c|}{ Prob $>F$} & $=$ & 0,0000 \\
\hline & & & & \multicolumn{2}{|c|}{ R-squared } & $=$ & 0,8507 \\
\hline & & & & \multicolumn{2}{|c|}{ Root MSE } & $=$ & 0,03559 \\
\hline ITC_CHI & Coef. & $\begin{array}{l}\text { Robust } \\
\text { Std. Err. }\end{array}$ & $\mathrm{t}$ & $P>|t|$ & $\begin{array}{l}\text { [95\% C } \\
\text { Inter }\end{array}$ & & \\
\hline ICOBRE & $-0,12986$ & 0,0137096 & $-9,47$ & 0,000 & $-0,156$ & 7501 & $-0,102962$ \\
\hline lEMBI_CHI & 0,032654 & 0,0071988 & 4,54 & 0,000 & 0,0185 & 3324 & 0,0467759 \\
\hline IDJ & 0,081204 & 0,0060346 & 13,46 & 0,000 & 0,0693 & 3659 & 0,0930419 \\
\hline IBROAD & 1,191962 & 0,0385339 & 30,93 & 0,000 & 1,116 & & 1,267553 \\
\hline _cons & 0,680261 & 0,2372189 & 2,87 & 0,004 & 0,214 & 9122 & 1,14561 \\
\hline
\end{tabular}

\section{COLOMBIA}

. Reg lTC_COL IPETROLEO IEMBI_COL IDJ IBROAD, r

\section{Linear regression}

$\begin{array}{llr}\text { Number of obs } & = & 1.384 \\ \mathrm{~F}(4,1379) & > & 2329,16 \\ \text { Prob }>\mathrm{F} & = & 0,0000 \\ \mathrm{R}-\text { squared } & = & 0,9082 \\ \text { Root MSE } & = & 0,03031\end{array}$

\begin{tabular}{|c|c|c|c|c|c|c|}
\hline ITC_COL & Coef. & $\begin{array}{c}\text { Robust } \\
\text { Std. Err. }\end{array}$ & $\mathrm{t}$ & $\mathrm{P}>|\mathrm{t}|$ & $\begin{array}{c}\text { [95\% Conf. } \\
\text { Interval] }\end{array}$ & \\
\hline ICOBRE & $-0,18688$ & 0,0076984 & $-24,28$ & 0,000 & $-0,2019859$ & $-0,171782$ \\
\hline IEMBI_COL & 0,123771 & 0,0044102 & 28,06 & 0,000 & 0,1151193 & 0,1324223 \\
\hline IDJ & 0,185792 & 0,0078288 & 23,73 & 0,000 & 0,1704345 & 0,2011498 \\
\hline IBROAD & 0,57446 & 0,0479594 & 11,98 & 0,000 & 0,4803783 & 0,6685408 \\
\hline Cons & 3,339785 & 0,1840334 & 18,15 & 0,000 & 2,97877 & 3,700801 \\
\hline
\end{tabular}

Fuente: elaboración propia. 